\title{
Synthesis of Highly Dispersed Small Metal-Organic Frameworks Crystal Using a Polymer Additive
}

\author{
Tak Hyeon Kim, Yang Sang Cho, and Chang Yeon Lee* \\ Department of Energy and Chemical Engineering, Incheon National University, Incheon 406-772, Korea \\ *E-mail: cylee@incheon.ac.kr \\ Received April 14, 2014, Accepted May 1, 2014
}

Key Words : Metal-organic frameworks (MOFs), BET surface area, Colloidal MOFs

Metal-organic frameworks (MOFs), which consist of organic building blocks and metal or metal-cluster secondary building units (SBU), have emerged as a promising alternative to conventional porous materials due to their ultra-high surface area, structural diversity, controllable pore sizes, and adjustable functionality. ${ }^{1}$ These distinct advantages of MOFs, as compared to other porous materials, have tremendous implications for potential applications such as gas and chemical storage ${ }^{2}$ separations, ${ }^{3}$ sensors ${ }^{4}$ light-harvesters ${ }^{5}$ drug delivery, ${ }^{6}$ and catalysis. ${ }^{7}$ Typically, the crystalline material of MOFs has been formed as a bulk crystal several hundred micrometers to several millimeters in size, with irregular morphologies. This may be not suitable for precise applications, including membrane separation, thin-film device formation, and applications in the biomedical field, all of which require small crystal sizes as well as uniform morphology of materials. Recently, several groups have developed a simple synthetic method for morphology controlled nano- and microscale MOFs by fine-tuning the nucleation and crystal growth rate. ${ }^{8-10}$

Co-MOF-74 $\left[\mathrm{Co}_{2}\right.$ (dihydroxyterephthalate(dhtp)) $]$ is a wellcharacterized MOF material containing high concentrations of open metal sites. It has been widely studied in the last decade due to its potential applications in carbon dioxide sequestration, ${ }^{11,12}$ hydrocarbon separations, ${ }^{13}$ and catalysis. ${ }^{14}$ The high density of open metal site in Co-MOF-74 plays a key role in its separation and catalytic performance. Conventional Co-MOF-74 has been synthesized as a large bulk crystal with irregular morphology and non-dispersive properties, which reduces the capability of Co-MOF-74 to be used for separation and catalysis applications. To overcome this limitation, we report on the formation of highly dispersed small Co-MOF-74 crystals controlled by the addition of ploy(vinylpyrrolidone) (PVP) during the formation process.

The micro-sized Co-MOF-74 crystal has been successfully synthesized by the simple solvothermal reaction of 2,5dihydroxyterephthalic acid (DHTA) and cobalt (II) nitrate hexahydrate (3:1 molar ratio) in the presence of varying

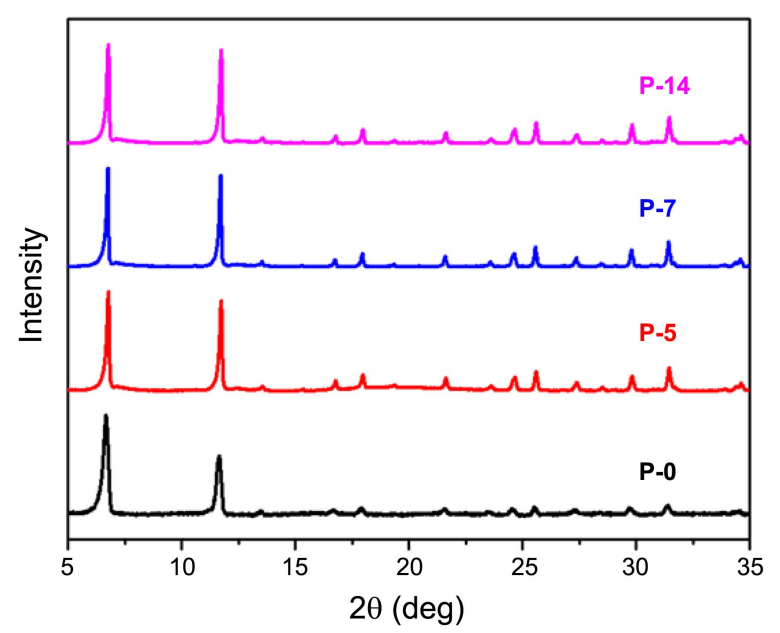

Figure 1. Powder X-ray diffraction (PXRD) for P-0, P-5, P-7, and P-14.

amounts of PVP $(5,7$, and $14 \mathrm{wt} \%$, denoted as P-5, P-7, and P-14 hereafter). Cobalt precursor, DHTA ligand, PVP, and $N, N$-dimethylformamide(DMF)/ethanol/water (1:1:1 volume $\mathrm{v}: \mathrm{v}: \mathrm{v})$ homogeneous mixtures were placed in a $20 \mathrm{~mL}$ vial with a screw cap. The reaction mixture was heated at $100{ }^{\circ} \mathrm{C}$ for $6 \mathrm{~h}$ with vigorous stirring. A fine orange solid was precipitated after $6 \mathrm{~h}$, and the dispersed solid was centrifuged and washed several times with both DMF and methanol. For the control experiment, we performed the same procedure as described above without the addition of PVP (denoted as P0 hereafter).

The powder X-ray diffraction (PXRD) data is depicted in Figure 1. PXRD patterns of all the as-synthesized samples were consistent with literature reported data and simulated patterns, ${ }^{11}$ which implied that appropriate MOF structures were synthesized. Interestingly, Co-MOF-74 synthesized in the presence of PVP (P-5, P-7, and P-14), showed superior crystallinity compared to the $\mathbf{P}-\mathbf{0}$ sample, with well-defined sharp peaks over the entire range of 2-theta values (Figure 1). Conversely, the control sample (P-0) exhibited only mode- 


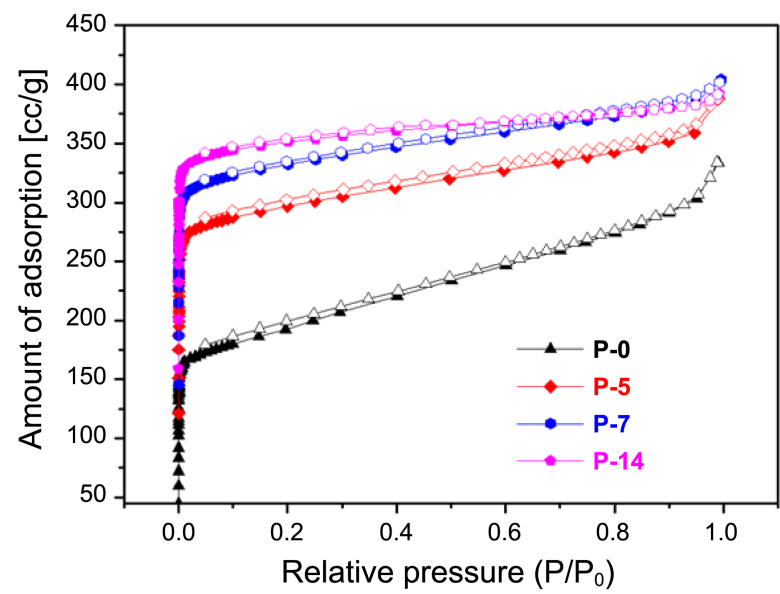

Figure 2. $\mathrm{N}_{2}$ isotherms at $77 \mathrm{~K}$ for $\mathbf{P - 0}, \mathbf{P}-\mathbf{5}, \mathbf{P}-\mathbf{7}$, and $\mathbf{P - 1 4}$.

rate crystallinity. Moreover, the relative intensity of the first two peaks of samples with added PVP were different from the first two peaks of the control sample, which indicated that PVP influenced the crystal growth direction.

The permanent porosity of all samples was confirmed by the measurement of nitrogen $\left(\mathrm{N}_{2}\right)$ adsorption-desorption isotherms (Figure 2). All isotherms displayed a type I isotherm, confirming their microporous nature. BrunauerEmmett-Teller (BET) surface areas of activated P-5, P-7, and P-14 were found to be 1183, 1329, $1428 \mathrm{~m}^{2} \mathrm{~g}^{-1}$, respectively, in excellent agreement with the reported Co-MOF-74 value ${ }^{13,14}\left(1327 \mathrm{~m}^{2} \mathrm{~g}^{-1}\right)$. The surface area of P-14 slightly exceeded the typical value. However, the BET surface area $\left(718 \mathrm{~m}^{2} \mathrm{~g}^{-1}\right)$ of $\mathbf{P}-\mathbf{0}$ was inferior to the typical Co-MOF-74 value. This result may imply that the structure formation of the control sample did not fully progress due to vigorous stirring during the formation. Co-MOF-74 is usually synthesized under solvothermal conditions without stirring for intact crystal growth. The surface areas of P-5, P-7, and P-14 increased with increasing PVP concentration; thus, proper MOF crystals were found to form when PVP was added in the solvothermal reaction mixture. Enhanced crystallinity on addition of PVP was observed during the PXRD experiment. (see above) From these findings, PVP was determined to play a key role in the formation of an intact MOF crystal by acting as a stabilizer.

Figure 3(3a-3d) shows scanning electron microscopy (SEM) images of all the samples. Each sample consisted of single-phase hexagonal column-structured particles with large aspect ratios. The traditional solvothermal synthesis of Co-MOF-74 affords a large crystal with a wide distribution of particle size and length, ranging from 100 to $200 \mu \mathrm{m}$ in length and from 5 up to $20 \mu \mathrm{m}$ in width. ${ }^{14,15}$ However, the average crystal size of the product prepared in this study was smaller than that of typical Co-MOF-74. This result indicated that fast nucleation was effectively achieved during production by vigorous stirring. Interestingly, crystal width and length increased with increasing PVP content, which implied that nucleation was delayed by PVP, though not diminished, and anisotropic crystal growth was likely to be
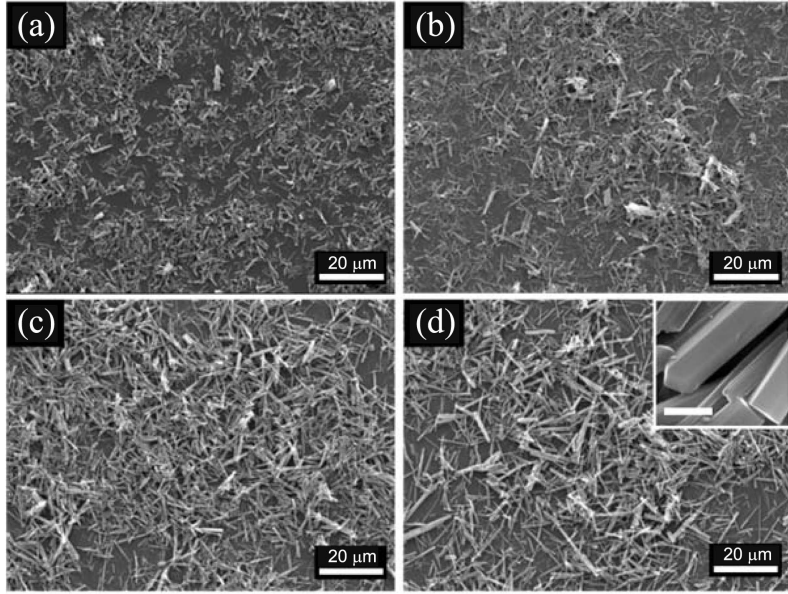

(e)
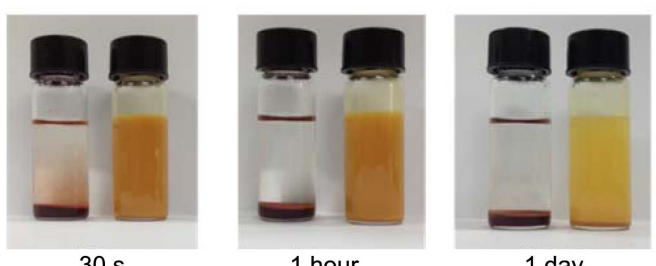

Figure 3. SEM images of MOFs: (a) P-0; (b) P-5; (c) P-7; (d) P14 (scale bar of inset: $1 \mu \mathrm{m}$ ) and (e) photograph of typical CoMOF-74 and P-14 suspension in $\mathrm{MeOH}$ after agitation.

induced by PVP addition. The pyrrolidone side chain in PVP preferentially interacts with metal ions and specific facets of the nanoparticles, which increments particle size and causes anisotropic growth. ${ }^{16}$ Yanai et al. recently reported that the MOF crystal size is increased in the presence of PVP, and observed that PVP acts as a weak capping ligand for decreasing the number of nucleation points. ${ }^{17}$ The increase in MOF crystal size with PVP, observed in this work, agrees with these past findings. Figure 3(e) shows the dispersion of the typical Co-MOF-74 and P-14 sample in methanol. Typical Co-MOF-74 rapidly settled to the bottom of the vial within $30 \mathrm{~s}$ after agitation, whereas $\mathbf{P - 1 4}$ was highly dispersed for over an hour and only fully settled after one day. Moreover, the micro-sized P-14 crystal was well dispersed in common

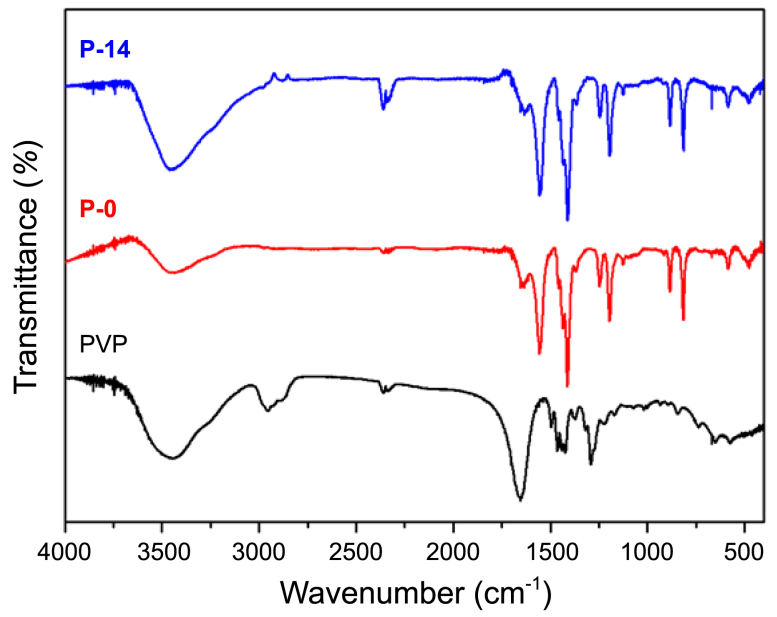

Figure 4. IR spectra of PVP, P-0, and P-14. 
solvents such as $N, N$-dimethylformamide (DMF), tetrahydrofuran (THF) and acetone.

Infrared spectroscopy was performed to investigate the existence of PVP in the P-14 sample and compare its spectrum to the spectrum of PVP. The P-0 IR spectrum was also obtained and compared with the P-14 spectrum. As shown in Figure 4, peaks near 2950 and $1291 \mathrm{~cm}^{-1}$, corresponding to $\mathrm{C}-\mathrm{H}$ and $\mathrm{C}-\mathrm{N}$ stretching of PVP, were not observed in P$\mathbf{1 4}$ and there were no differences in the IR spectra of $\mathbf{P - 0}$ and P-14. These results indicate that PVP was completely removed from the MOF crystal during the washing process. Therefore, PVP can be used to manipulate the characteristic properties of MOFs without any contamination of the final product.

In conclusion, we have successfully synthesized microsized intact Co-MOF-74 crystals by using PVP-assisted solvothermal reactions. The newly synthesized small CoMOF-74 was easily dispersed in common solvents, which will allow us to extend MOF's applications into more precise product areas, such as membrane-based separation, thin film device and catalysis, which are limited by non-dispersive characteristics of MOFs.

\section{Experimental}

Ethanol, DMF, 2,5-dihydroxyterephthalic acid, cobalt(II) nitrate hexahydrate, and polyvinylpyrrolidone (PVP, $\mathrm{M}_{\mathrm{w}} \approx$ $55,000)$ were purchased from Sigma-Aldrich and used as received. Co-MOF-74 was synthesized according to a published procedure. ${ }^{11}$ SEM images were obtained from a FE-SEM (JEOL, JSM-7001F) operated at an acceleration voltage of $10 \mathrm{kV}$. Samples were coated by a layer of $\mathrm{Au}-\mathrm{Pt}$ alloys ( $\sim 3 \mathrm{~nm}$ thickness) prior to imaging. Powder X-ray diffraction (PXRD) patterns were recorded on a Rigaku XDS 2000 diffractometer using nickel-filtered copper $\mathrm{K} \alpha$ radiation $(\lambda=1.5412 \AA)$ over a range of $3^{\circ}<2 \theta<50^{\circ}$ in $0.1^{\circ}$ steps with a $1 \mathrm{~s}$ counting time per step. Infrared spectra were obtained from a Varian 640-IR spectrophotometer.

Synthesis of P-0, P-5, P-7, and P-14. 2,5-Dihydroxyterephthalic acid (DHTA) $(0.0362 \mathrm{~g}, 0.182 \mathrm{mmol}), \mathrm{Co}\left(\mathrm{NO}_{3}\right)_{2} \cdot 6 \mathrm{H}_{2} \mathrm{O}$ (0.178 g, $0.613 \mathrm{mmol})$, PVP (0 g for P-0, $0.608 \mathrm{~g}$ for P-5, $1.014 \mathrm{~g}$ for P-7, $2.028 \mathrm{~g}$ for P-14), and $15 \mathrm{~mL}$ of DMF: ethanol:water (1:1:1, v:v:v) were combined in a $20 \mathrm{~mL}$ vial equipped with a magnetic stir bar. The vial was then capped with a Teflon-lined cap and sonicated for 5 min to obtain a homogeneous solution. The resulting reaction mixture was placed in a $100{ }^{\circ} \mathrm{C}$ oil bath and vigorously stirred (300 rpm). After $6 \mathrm{~h}$, orange powder was precipitated from the reaction mixture. The resulting suspension was cooled to room temperature. Finally, the sample was washed twice with DMF $(40 \mathrm{~mL})$ and twice with $\mathrm{MeOH}(40 \mathrm{~mL})$ to remove excess PVP and centrifuged at $2500 \mathrm{rpm}$. The remaining orange powder was then stored in $\mathrm{MeOH}$ until further use.

$\mathbf{N}_{2}$ Sorption Isotherms. $\mathrm{N}_{2}$ adsorption/desorption isotherms were measured volumetrically at $77 \mathrm{~K}$ in the range $7.0 \times 10^{-6} \leq P / P_{0} \leq 1.00$ with an Autosorb-iQ outfitted with the micropore option by Quantachrome Instruments (Boynton Beach, Florida USA), running the Autosorb-iQ Win software package. After solvent exchange of the as-synthesized materials with $\mathrm{MeOH}(2 \times 10 \mathrm{~mL}, 12 \mathrm{~h}$ each time $)$, all samples were activated (i.e., outgassed) at $250{ }^{\circ} \mathrm{C}$ for $5 \mathrm{~h}$ by using the outgas port of the Autosorb-iQ instrument. The specific surface areas for $\mathrm{N}_{2}$ were calculated using the BrunauerEmmett-Teller (BET) model in the linear range, determined using the consistency criteria.

Acknowledgments. This research was supported by Basic Science Research Program through the National Research Foundation of Korea (NRF) funded by the Ministry of Science, ICT \& Future Planning (NRF-2013R1A1A1058839).

\section{References}

1. Férey, G. Chem. Soc. Rev. 2008, 37, 191.

2. Suh, M. P.; Park, H. J.; Prasad, T. K.; Lim, D.-W. Chem. Rev. 2012, 112, 782 .

3. Li, J.-R.; Sculley, J.; Zhou, H.-C. Chem. Rev. 2012, 112, 869.

4. Kreno, L. E.; Leong, K.; Farha, O. K.; Allendorf, M.; Van Duyne, R. P.; Hupp, J. T. Chem. Rev. 2012, 112, 1105.

5. Lee, C. Y.; Farha, O. K.; Hong, B. J.; Sarjeant, A. A.; Nguyen, S. T.; Hupp, J. T. J. Am. Chem. Soc. 2011, 133, 15858.

6. Horcajada, P.; Gref, R.; Baati, T.; Allan, P. K.; Maurin, G.; Couvreur, P.; Férey, G.; Morris, R. E.; Serre, C. Chem. Rev. 2012, $112,1232$.

7. Lee, J.; Farha, O. K.; Roberts, J.; Scheidt, K. A.; Nguyen, S. T.; Hupp, J. T. Chem. Soc. Rev. 2009, 38, 1450.

8. Sindoro, M.; Yanai, N.; Jee, A.-Y.; Granick, S. Acc. Chem. Res. 2013, ASAP.

9. Ranft, A.; Betzler, S. B.; Haase, F.; Lotsch, B. V. CrystEngComm 2013, 15, 9296.

10. Cho, W.; Lee, H. J.; Oh, M. J. Am. Chem. Soc. 2008, 130, 16943.

11. Caskey, S. R.; Wong-Foy, A. G.; Matzger, A. J. J. Am. Chem. Soc. 2008, 130, 10870.

12. McDonald, T. M.; Lee, W. R.; Mason, J. A.; Wiers, B. M.; Hong, C. S.; Long, J. R. J. Am. Chem. Soc. 2012, 134, 7056.

13. Bae, Y.-S.; Lee, C. Y.; Kim, K. C.; Farha, O. K.; Nickias, P.; Hupp, J. T.; Nguyen, S. T.; Snurr, R. Q. Angew. Chem. Int. Ed. 2012, 51, 1857.

14. Cho, H.-Y.; Yang, D.-A.; Kim, J.; Jeong, S.-Y.; Ahn, W.-S. Catal. Today 2012, 185, 35.

15. Chmelik, C.; Mundstock, A.; Dietzel, P. D. C.; Caro, J. Microporous Mesoporous Mater. 2014, 183, 117.

16. Lee, S. J.; Park, G.; Seo, D.; Ka, D.; Kim, S. Y.; Chung, I. S.; Song, H. Chemistry - A European Journal 2011, 17, 8466.

17. Yanai, N.; Sindoro, M.; Yan, J.; Granick, S. J. Am. Chem. Soc. 2012, $135,34$. 